\title{
A Questão da Propriedade Intelectual no Centro de Incubação e Desenvolvimento Empresarial (CIDE)
}

\author{
The Issue of Intellectual Property at the Center for Incubation and \\ Business Development
}

\author{
Darlene Silveira Rodrigues ${ }^{1}$ \\ Hyrmir Alexandre Sousa ${ }^{1}$ \\ Raimundo Correa de Oliveira ${ }^{1}$ \\ ${ }^{1}$ Universidade do Estado do Amazonas, Manaus, AM, Brasil
}

\begin{abstract}
Resumo
Este trabalho tem como questão principal verificar as ações desenvolvidas pela incubadora do CIDE quanto à proteção da propriedade intelectual, portanto, pretende observar se, em meio a esse processo de incubação, as empresas estariam preocupadas em proteger esses ativos. Foram empregados como métodos de pesquisa o bibliográfico, documental e o de campo. De acordo com a pesquisa realizada e de todos os pontos levantados, pode-se concluir que a equipe da incubadora $e$ as das empresas residentes devem ser qualificadas para tratar dos ativos de PI desenvolvidos durante o processo de incubação, criando assim um ambiente favorável para proteção $e$ transferência de tecnologia. Transferência de tecnologia é o meio pelo qual um conjunto de conhecimentos, habilidades e procedimentos aplicáveis aos problemas da produção são transferidos, por transação de caráter econômico ou não, de uma organização a outra, ampliando a capacidade de inovação da organização receptora.
\end{abstract}

Palavras-chave: Incubadoras. Marcas. Propriedade Intelectual. Ativos.

\begin{abstract}
This work has as main question to verify the actions developed by the CIDE incubator regarding the protection of intellectual property. Therefore, to check whether in the midst of this incubation process, companies would be concerned about protecting these assets. Thus, the general objective of this article is to identify the importance of trademark protection as a competitive strategy for micro and small companies. The method of bibliographical, documentary and field research was used. According to the research carried out and all points raised, it can be concluded that the incubator team and the resident companies should be qualified to handle the IP assets developed during the incubation process and thus create a favorable environment for protection and technology transfer. Technology transfer is the means by which a set of knowledge, skills and procedures applicable to the problems of production are transferred, by economic transaction or not, from one organization to another, increasing the capacity of innovation of the receiving organization.
\end{abstract}

Keywords: Incubators. Brands. Intellectual Property. Intagible assets.

Área Tecnológica: Software. Incubadora. Ativos Intangíveis. 


\section{Introdução}

A Associação Nacional de Entidades Promotoras de Empreendimentos Inovadores (ANPROTEC, 2012) define que incubadora é uma entidade que tem por objetivo oferecer suporte a empreendedores para que eles possam desenvolver ideias inovadoras e transformá-las em empreendimentos de sucesso. Para isso, oferece infraestrutura, capacitação e suporte gerencial, orientando os empreendedores sobre aspectos administrativos, comerciais, financeiros e jurídicos, entre outras questões essenciais ao desenvolvimento de uma empresa.

A Associação Brasileira de Startups afirma que a incubadora de empresas é uma forma de estimular o empreendedorismo, pois fortalece e prepara as pequenas empresas com o intuito de fazê-las sobreviver no mercado. É um local que abriga esses negócios, oferecendo estrutura capaz de estimular, fornecer e agilizar a transferência de resultados de pesquisa para atividades voltadas à produção.

Segundo a Lei de Inovação, inciso III-A, tem-se o conceito a seguir.

Artigo $2^{\circ}$ - Para os efeitos desta Lei, considera-se:

[...]

III-A - incubadora de empresas: organização ou estrutura que objetiva estimular ou prestar apoio logístico, gerencial e tecnológico ao empreendedorismo inovador e intensivo em conhecimento, com o objetivo de facilitar a criação e o desenvolvimento de empresas que tenham como diferencial a realização de atividades voltadas à inovação [... ]. (BRASIL, 2004, art. $2^{\circ}$ )

Ao se falar em inovação tem-se que a proteção dos ativos de propriedade intelectual desenvolvida pelas empresas, incubadas ou não, pode se tornar atividade necessária e primordial para a continuidade do crescimento das firmas, já que garante segurança jurídica contra imitação e outras atividades ilícitas. Além disso, a propriedade intelectual está entre um dos mecanismos de apropriabilidade de tecnologias e conhecimento (SILVA, 2016).

Segundo a ANPROTEC (2012), existem diversos tipos de incubadoras: as de base tecnológica (abrigam empreendimentos que utilizam tecnologias); as tradicionais (dão suporte a empresas de setores tradicionais da economia); as mistas (aceitam tanto empreendimentos de base tecnológica quanto de setores tradicionais) e as sociais (que têm como público-alvo cooperativas e associações populares).

A escolha do tema foi ao encontro dos interesses de investigar e conhecer sobre a importância da Propriedade Intelectual nas empresas que fazem parte do Centro de Incubação e Desenvolvimento Empresarial (CIDE).

Portanto, em meio a esse processo de incubação, estariam as empresas preocupadas em proteger esses ativos? Dessa forma, o objetivo geral deste artigo é analisar as ações desenvolvidas pela Incubadora do CIDE quanto à proteção da propriedade intelectual.

A justificativa de escolha do tema se deu devido à necessidade de verificar se as empresas incubadas estão tendo a preocupação do registro de suas marcas por meio do apoio e conhecimento obtido pela incubadora. 
Segundo o INPI (2013), a marca é um sinal distintivo cujas funções principais são identificar a origem e distinguir produtos ou serviços de outros idênticos, semelhantes ou afins de origem diversa.

De acordo com a legislação brasileira, são passíveis de registro como marca todos os sinais distintivos visualmente perceptíveis, não compreendidos nas proibições legais, conforme disposto no artigo 122 da Lei n. 9.279/96 (Lei da Propriedade Industrial).

Portanto, para melhor esclarecer a questão é necessário descrever os objetivos específicos, como: identificar se o CIDE possui práticas de gestão da propriedade intelectual, próprias ou em parceira, em benefício das empresas incubadas; apontar o grau de importância socioeconômica da proteção do direito dos ativos intangíveis da propriedade intelectual; citar a importância de uma cultura de proteção das marcas; averiguar se as empresas do CIDE estão protegendo suas marcas através do registro. Identificar e discutir as principais vantagens e desvantagens.

\section{Metodologia}

Para o alcance dos objetivos propostos, este trabalho fundamenta-se, principalmente, na pesquisa qualitativa, que é aquela que busca conhecer os fenômenos sociais por meio dos significados que estes têm para as pessoas. Desse modo foram utilizados os seguintes tipos de pesquisa:

a) Pesquisa bibliográfica: bases de dados SciELO, Periódicos Capes, Portal do CIDE.

b) Pesquisa documental: bases de dados do INPI.

c) Pesquisa de campo: Entrevistas com o gerente do CIDE, em que foram levantadas informações com relação ao número de empresas incubadas e graduadas, bem como se tais empreendimentos possuem depósito de pedido de registro de marca. O questionário contém perguntas específicas sobre a incubadora e de que maneira esta trata o tema propriedade intelectual nas suas atividades, além de levantar informações quanto à identificação da incubadora, como: Estado, sede, nome, entidade mantenedora. Procurou-se saber o ano em que a incubadora iniciou sua operação, considerando-se o momento no qual ela incubou o seu primeiro empreendimento. Identificou-se também o foco da incubadora, conforme as classificações: mistas, tecnológicas e tradicionais. Também foram obtidas informações sobre o estágio em que as incubadoras se encontram com relação à implantação do Modelo CERNE.

Na pesquisa de campo, houve dificuldade quanto ao acesso a informações, fossem elas obtidas em documentos internos ou por entrevistas.

\subsection{Inovação}

Inovação é uma combinação de necessidades sociais e de demandas do mercado com os meios científicos e tecnológicos para resolvê-las. A inovação tecnológica é entendida aqui como a transformação do conhecimento em produtos, processos e serviços que possam ser colocados no mercado. (CARON, 2004). 
Diante disso, a expressão inovação tecnológica vem ganhando cada vez mais importância de um modo generalizado, nas últimas décadas, no âmbito empresarial. A explicação para isso é bem fácil, visto que uma organização preza por bons resultados, se está inserida no mundo tecnológico atual no qual se vive.

Conforme estabelece o Manual de Oslo, inovação é:

[...] a implementação de um produto (bem ou serviço) novo ou significativamente melhorado, ou um novo processo, ou um novo método de marketing, ou um novo método organizacional nas práticas de negócios, na organização do local de trabalho ou nas relações externas (OCDE, 2005).

Há quatro tipos de inovação que abrangem as mudanças nas atividades das empresas, segundo a OCDE (2005):

a) Inovações em produtos: é a introdução de um bem ou serviço novo ou significativamente melhorado. Essa inovação tanto pode incorporar novos conhecimentos ou tecnologias ou fazer combinações com esses mesmos elementos.

b) Inovações em marketing: envolvem implementações de novos métodos de marketing. Essas mudanças estão voltadas para atender às necessidades do mercado consumidor, portanto, abrangem modificações na aparência do produto, divulgação, distribuição, preços e benefícios.

c) Inovações Organizacionais: implementação de um novo método organizacional nas práticas de negócios da empresa, envolvendo situações de redução de custos, local de trabalho, relações externas e outras que podem favorecer a tomada de decisão.

d) Uma inovação de processo é a implementação de um método de produção ou distribuição novo ou significativamente melhorado. Incluem-se mudanças significativas em técnicas, equipamentos e/ou softwares.

Da interação entre universidades, empresas e governos, surgiram os chamados mecanismos para inovação, que compreendem incubadoras de empresas, aceleradoras de negócios, parques e polos científicos, tecnológicos e de inovação, tecnópolis e similares (ANPROTEC, 2012). Tais mecanismos são atualmente chamados de habitats de inovação, por estarem em constante transformação (SPOLIDORO et al., 2014).

Para Spolidoro et al. (2014), os ambientes de inovação têm por objetivo geral criar a capacidade local e regional sustentável para a inovação em todos os domínios das atividades humanas, contribuindo para a construção de um processo de desenvolvimento local e regional sustentável, socialmente responsável e competitivo da economia globalizada.

Vários são esses espaços considerados como ambientes de inovação, áreas de inovação ou habitats de inovação, e, segundo Spolidoro et al. (2014, p. 9), pode-se listar como exemplo: Tecnopolos, Smartcities, Redes de Inovação, Parque Tecnológico, Parque Empresarial, Parque de Inovação, Parque Científico e Tecnológico, Núcleos de Inovação, Centros de Negócios e Inovação, Aceleradoras de Empresas, Hotéis de Projeto e Incubadoras de Empresas. 


\subsection{As Incubadoras de Empresas e a Propriedade Intelectual}

Segundo a ANPROTEC (2012), as incubadoras de empresa começaram a ser criadas a partir de uma iniciativa do CNPq, na década de 1980, de implantação do primeiro Programa de Parques Tecnológicos no País. Essa iniciativa, que semeou a noção de empreendedorismo inovador no Brasil, desencadeou o surgimento de um dos maiores sistemas mundiais de incubação de empresas. Diversas incubadoras também se tornaram o embrião de parques tecnológicos quando o ambiente brasileiro se tornou mais sensível à inovação. Inicialmente, as incubadoras estavam focadas apenas em setores intensivos em conhecimentos científicos-tecnológicos, como informática, biotecnologia e automação industrial. Habitualmente denominadas incubadoras de empresas de base tecnológica, ou incubadoras tecnológicas, tinham como propósito, assim, a criação de empresas com potencial para levar ao mercado novas ideais e tendências tecnológicas. Atualmente, além do objetivo inicial, elas têm o propósito de contribuir para o desenvolvimento local e setorial.

Incubadora de empresas é uma organização ou estrutura que objetiva estimular ou prestar apoio logístico, gerencial e tecnológico ao empreendedorismo inovador e intensivo em conhecimento, com o objetivo de facilitar a criação e o desenvolvimento de empresas que tenham como diferencial a realização de atividades voltadas à inovação (BRASIL, 2016).

Contudo, o principal objetivo de uma incubadora de empresas deve ser a produção de empresas de sucesso, em constante desenvolvimento, financeiramente viáveis e competitivas em seu mercado, mesmo após deixarem a incubadora, em geral, em um prazo de dois a quatro anos. Ou seja, todo empreendedor e sua empresa passam por um processo de seleção para serem admitidos em uma incubadora, devendo apresentar, entre outros documentos, um plano de negócios do empreendimento (DORNELAS, 2016, p. 205).

Para Dornelas (2016, p. 205), a empresa incubada não encontrará fora da incubadora as facilidades existentes dentro dela, a preços tão competitivos e de forma tão integrada, por isso, a taxa de mortalidade de empresas incubadas é muito menor que a das micro e pequenas empresas em geral, e a procura por vagas em incubadoras, por parte das empresas nascentes, vem aumentando no país.

A empresa pode ser incubada residente (quando ocupa um espaço dentro do prédio da incubadora) ou incubada não residente (caso em que tem sua própria sede, mas recebe suporte da incubadora). O tempo médio de incubação de uma empresa é de três anos, prazo que, no entanto, varia de acordo com as características do empreendimento. Empresas da área de Tecnologia da Informação e Comunicação tendem a ficar menos tempo incubadas do que empreendimentos do setor de Biotecnologia, por exemplo, já que trabalham com tecnologias que possuem um ciclo de desenvolvimento mais curto. $\mathrm{O}$ importante é que o empreendimento, ao se graduar, esteja preparado para o mercado. Durante o processo de incubação, as incubadoras realizam acompanhamentos periódicos para avaliar o nível de desenvolvimento das empresas (ANPROTEC, 2012).

Em se tratando de empresa graduada, a ANPROTEC (2012) define que é uma empresa que já passou pelo processo de incubação, ou seja, que recebeu suporte de uma incubadora $e$ já possui competências suficientes para se desenvolver sozinha. A empresa, depois de graduada, pode continuar sendo associada à incubadora, mas não pode mais residir no espaço físico da instituição. 
Para Spolidoro (1999), incubadora é um ambiente que favorece a criação e o desenvolvimento de empresas e de produtos (bens e serviços), em especial aqueles inovadores e intensivos de conteúdo intelectual (produtos onde o custo da parcela do trabalho intelectual é maior que o da parcela devida a todos os demais insumos).

Nesse sentido, os ativos intangíveis, que formam o capital intelectual de uma empresa (tecnologia da informação, marca e conhecimentos resultantes dos projetos de pesquisa e desenvolvimento) desempenham um importante papel na criação de valor para o negócio, uma vez que são únicos, criam competências exclusivas e produzem resultados melhores (KRAEMER, 2004).

Partindo-se do princípio de que incubadoras de empresas recebem empreendimentos inovadores, nos diversos setores da economia, é indispensável que a incubadora e as próprias empresas considerem a proteção e a gestão da propriedade intelectual essenciais para o desenvolvimento de seus negócios (SILVA, 2016, p. 43).

Ao contrário dos ativos tangíveis com os quais os empresários e contadores estão familiarizados (propriedade, fábricas, equipamentos e dinheiro), os ativos intangíveis são aqueles que não possuem existência física. São difíceis de serem identificados, de serem distribuídos e avaliados, de forma eficaz. Porém, uma vez descobertos e explorados, possibilitam vantagem competitiva (CARVALHO; SOUZA, 1999).

A convenção da Organização Mundial da Propriedade Intelectual (OMPI, 2002) define como propriedade intelectual: a soma dos direitos relativos às obras literárias, artísticas e científicas, às interpretações dos artistas intérpretes e às execuções dos artistas executantes, aos fonogramas $e$ às emissões de radiodifusão, às invenções em todos os domínios da atividade humana, às descobertas científicas, aos desenhos e modelos industriais, às marcas industriais, comerciais e de serviço, bem como às firmas comerciais e denominações comerciais, à proteção contra a concorrência desleal e a todos os outros inerentes à atividade intelectual nos domínios industrial, científico, literário e artístico.

A difusão dos conceitos e a utilização dos instrumentos de proteção da propriedade intelectual são fundamentais para empresas e indivíduos assegurarem que suas criações, invenções, obras artísticas ou literárias tenham retorno financeiro quando da atividade de comercialização desses ativos. Além disso, dentro do contexto da era da economia do conhecimento, a propriedade intelectual legalmente protegida transformou-se em um importante ativo para a competitividade das empresas que desejam otimizar o valor desses bens (JUNGMANN; BONETTI, 2010).

Segundo Jungmann e Bonetti (2010), para uma empresa atingir seu objetivo é necessário saber como planejar a estratégia do negócio, como proteger produtos atuais e futuros, gerando valor para sua capacidade inovativa. Este processo é de grande valia no desenvolvimento das nações, na medida em que tem como finalidade:
a) favorecer o comércio internacional;
b) estimular novos métodos de produção;
c) aumentar a produtividade;
d) gerar riquezas;
e) melhorar a qualidade de vida; 
f) fomentar a faculdade criadora;

g) aumentar as possibilidades da ciência e da tecnologia;

h) enriquecer o mundo da literatura e das artes.

Em relação à proteção, dependendo da estratégia comercial da empresa, o mesmo produto poderá dispor de vários tipos de proteção, que cobrem diferentes aspectos, pela utilização apropriada dos instrumentos da propriedade intelectual. Por sua característica de identificar e diferenciar produtos, a marca torna-se uma das formas de proteção mais importante e aplicável no mundo empresarial (JUNGMANN; BONETTI, 2010).

Usar opções distintas para proteção de um produto garante um diferencial competitivo ainda mais forte, porque a empresa dispõe de diferentes tipos de direitos de exclusividade sobe aquele bem. Assim, ela poderá impedir terceiros, em todos os territórios em que dispõe de proteção, de copiar, produzir, usar, colocar à venda, vender, importar e exportar seu produto sem o seu consentimento.

Nesse sentido, será dada ênfase nesta pesquisa para a importância do registro de uma marca para a proteção e desenvolvimento de uma incubadora de empresa.

\subsection{Marcas}

Marca é um sinal distintivo cujas funções principais são identificar a origem e distinguir produtos ou serviços de outros idênticos, semelhantes ou afins de origem diversa (INPI, 2013). Uma marca só pode ser requerida por pessoa física ou jurídica que exerça atividade lícita, efetiva e compatível com o produto ou serviço que a marca visa a distinguir.

$\mathrm{O}$ registro de marca destina-se à proteção de produtos e serviços. A marca, para ser registrada, precisa ser distintiva, isto é, ser diferente o suficiente para ser capaz de identificar - sem ambiguidades - produtos ou serviços de outros semelhantes.

É possível citar algumas vantagens de se registrar uma marca: possibilita que os consumidores diferenciem produtos semelhantes; permite que as empresas promovam seu portfólio de produtos e serviços; é importante para a comercialização e é a base para o estabelecimento da imagem e reputação de uma linha de produtos no mercado; pode ser licenciada e fornecer uma fonte alternativa de receita por meio de pagamento de royalties; é um elemento fundamental nos acordos de franquia; pode ser um ativo comercial de valor; incentiva os empresários a investirem na manutenção ou no aprimoramento da qualidade dos seus produtos; pode ser útil para a obtenção de financiamentos (INPI, 2013).

\subsection{Incubadora do Centro de Incubação e Desenvolvimento Empresarial (CIDE)}

O Centro de Incubação e Desenvolvimento Empresarial (CIDE) tem a missão de estruturar a criação e o desenvolvimento de empresas inovadoras de base tecnológica com ênfase nos setores de biotecnologia, tecnologia da informação e eletrônica através de ações que contribuam para incentivar o empreendedor e o desenvolvimento socioeconômico do Estado.

Para atingir suas finalidades, o CIDE utiliza o sistema de incubação de empresas, fazendo ponte entre o mercado e o desenvolvimento tecnológico, respeitando as características próprias 
da região. As áreas de atuação das incubadas são: Software; Biojoias; Alimentos; Cosméticos; Fitoterápicos; Fitocosméticos; Laboratórios de análises; Medicamentos injetáveis; e reciclagem de materiais.

\subsection{Histórico}

Primeira incubadora de empresas do Estado do Amazonas (PIONEIRA). Durante o ano de 1998, o Instituto Euvaldo Lodi (IEL), regional do Amazonas, sob a direção de um empresário local, sr. Moysés Israel, tomou a iniciativa de iniciar um processo de sensibilização junto à comunidade da região visando a implantação de uma incubadora de empresas em Manaus. Esse processo consistiu de apresentações junto a dirigentes de diversas entidades da cidade, representativas de setores do governo, da academia, de pesquisas e do empresariado em geral. Participaram dessa etapa de sensibilização representantes do Instituto Euvaldo Lodi Nacional, da Associação Nacional de Entidades Promotoras de Empreendimentos Inovadores (ANPROTEC) e de incubadoras já constituídas, como o Centro Empresarial para Laboração de Tecnologias Avançadas (CELTA), vinculado à Fundação Centros de Referência em Tecnologias Inovadoras (CERTI), de Florianópolis. Além dessas apresentações e dos debates realizados com o apoio da Federação das Indústrias do Estado do Amazonas (FIEAM), foram realizadas também visitas técnicas a incubadoras de outros Estados, quando foram mostradas experiências de empreendimentos em operação.

Paralelamente a essas iniciativas, o IEL/AM iniciou a busca por um local para a instalação do empreendimento, quando foi localizado um imóvel que havia pertencido a um extinto órgão do Governo Federal, denominado Fundação Projeto Rondon, sem utilização naquele momento e que estava sob a responsabilidade da Superintendência da Zona Franca de Manaus (SUFRAMA). Após negociações entre o IEL/AM, a FIEAM e a SUFRAMA, esta assinou um Convênio com o IEL/AM de cessão de uso do imóvel, com o objeto específico de implantação do programa de incubadoras de empresas. Deu-se início às atividades, com o lançamento da pedra fundamental, em vinte de maio de 1999. Na época, um dos incentivadores na implantação do CIDE foi o presidente da FIEAM, José Nasser, e o Diretor Regional do IEL/AM, Moisés Benarrós Israel. Foi elaborado um estatuto do CIDE, aprovado em 20 de outubro de 1999, e criado o Conselho Administrativo em 27 de janeiro de 2000. Sua inauguração aconteceu em 19 de maio de 2000.

\section{Resultados Obtidos}

Observou-se que a incubadora do CIDE tem como foco o desenvolvimento de empresas inovadoras de base tecnológica com ênfase nos setores de biotecnologia, tecnologia da informação e eletrônica. As áreas de atuação das incubadas são: Software; Biojoias; Alimentos; Cosméticos; Fitoterápicos; Fitocosméticos; Laboratórios de análises; Medicamentos injetáveis; e reciclagem de materiais.

Quanto à certificação do modelo CERNE, a incubadora está em fase de implantação. Constatou-se que o CIDE tem algumas parcerias com instituições como Senai, Fieam, Sebrae e até a própria Suframa. 
Verificou-se que o CIDE está com 22 empresas incubadas residentes, 12 delas empresas no ramo de tecnologia e outras dez nas outras áreas: três empresas de cosméticos fitoterápicos, quatro de alimentos, uma empresa de laboratório de análise, uma de medicamentos injetáveis e reciclagem de materiais, e uma empresa de serigrafia.

A partir da pesquisa realizada na base de dados gratuita do INPI, foi possível verificar as empresas que realizaram o pedido de registro de marca, as empresas que tiveram seus pedidos de registro de marca indeferidos, as empresas que não realizaram o pedido de registro e as que estão com os registros extintos.

Gráfico 1 - Situação dos Pedidos dos Registros de Marcas

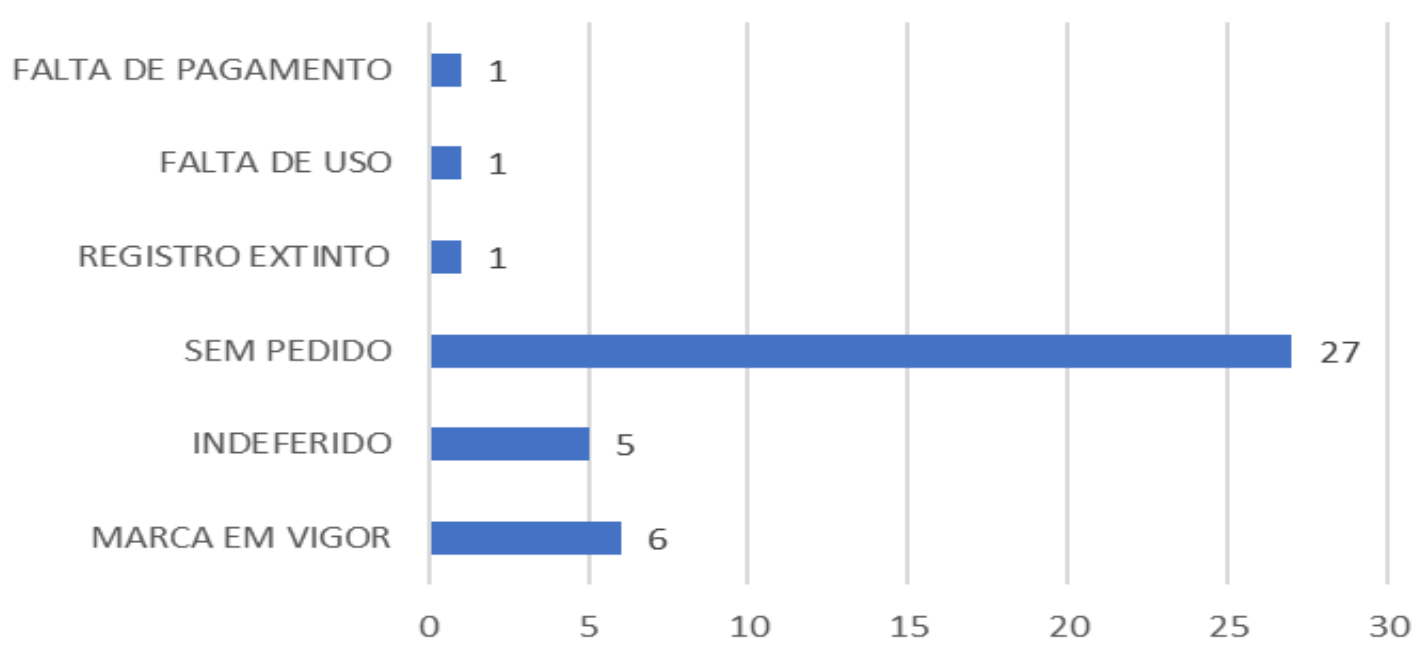

Fonte: Elaborado pelos autores deste artigo (2019)

Já com relação às empresas incubadas, foi verificado que o CIDE possui 22 empresas residentes, 75 graduadas, cinco associadas e duas startups.

Gráfico 2 - Como estão distribuídas as Empresas no CIDE
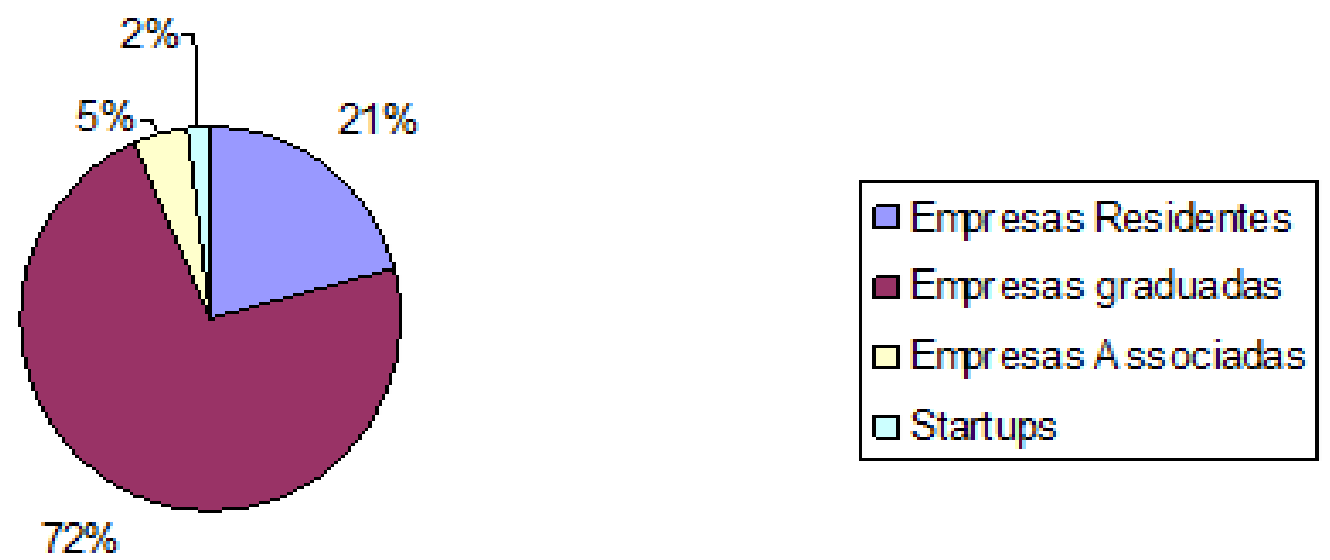

Fonte: Elaborado pelos autores deste artigo (2019) 


\section{Considerações Finais e Recomendações}

Diante dos conceitos abordados sobre inovação, incubadoras e propriedade intelectual, é possível afirmar que a pesquisa em questão mostrou alguns dos aspectos que envolvem a relação entre os ativos intangíveis, a propriedade intelectual e a incubadora. Dessa forma, o estudo proporcionou um conhecimento mais profundo em relação à realidade das empresas que participam desse modelo de negócios, o qual busca favorecer o desenvolvimento e a maturação e fortalecimento das organizações residentes, graduadas e associadas. Da propriedade intelectual, optou-se em estudar sobre o registro de marcas, especificamente os pedidos de registros realizados pelas empresas incubadas no Centro de Incubação e Desenvolvimento Empresarial (CIDE).

Buscou-se levantar dados concretos - com relação à proteção intelectual dos ativos intangíveis através da base de dados gratuita do INPI - dessas empresas incubadas e graduadas. Ao todo são 75 empresas (graduadas, incubadas, associadas), porém só foi possível identificar 41 empresas, número que inclui as residentes, graduadas e associadas. A ausência de informações mais detalhadas, por parte da incubadora e das empresas, com relação às empresas graduadas, dificultou mensurar a realidade desses empreendimentos, principalmente quanto aos ativos de PI gerados, bem como identificar se essas empresas que passaram pelo processo de incubação ainda estão atuando.

Em relação à gama de ativos de PI, optou-se por quantificar indicadores relacionados a marca, gerados tanto por empreendimentos incubados como por graduados e associados. De posse dos dados levantados, foi possível identificar que, das quarenta e uma empresas pesquisadas, apenas 14 empresas realizaram o pedido de registro da marca. Foi observado que cinco pedidos foram indeferidos e seis estão em vigor, um está extinto e os outros dois estão extintos por falta de uso e falta de pagamento das taxas.

Não foi possível obter dados atualizados com relação ao número de empregos gerados, mas foi possível identificar que em 2015 a incubadora gerou 314 empregos diretos na região, conforme informação de seu presidente executivo. Não foi possível obter dados também sobre o faturamento desses empreendimentos e se os negócios ainda estão ativos, de maneira a permitir mensurar a taxa de mortalidade dos negócios pós incubação.

Constatou-se que as participações em feiras nacionais e internacionais aumentam as oportunidades de novos negócios e incentivam a atenção da proteção dos ativos intangíveis das marcas, e que, por conta das dificuldades encontradas em registrar seus ativos, poucas empresas possuem o registro e outras estão em processo.

Em relação à propriedade intelectual, identificou-se que os gestores do CIDE têm criado iniciativas para capacitar e apoiar os empresários a investir nos seus ativos de propriedade intelectual, mas a iniciativa deve partir da gestão de cada empresa incubada. Desse modo, não teriam como discutir a proteção de tais ativos sem conhecerem os requisitos necessários para incentivar e proteger. Intimamente ligado à capacitação, se encontra o incentivo financeiro para o contínuo desenvolvimento das incubadoras.

De acordo com a pesquisa realizada $e$ de todos os pontos levantados, pode-se concluir que as equipes da incubadora e das empresas residentes devem ser qualificadas para tratar dos ativos de PI desenvolvidos durante o processo de incubação; e assim se cria um ambiente 
favorável à proteção e transferência de tecnologia. Transferência de tecnologia é o meio através do qual um conjunto de conhecimentos, habilidades e procedimentos aplicáveis aos problemas da produção são transferidos, por transação de caráter econômico ou não, de uma organização a outra, ampliando a capacidade de inovação da organização receptora.

Sugere-se a exigência de que as incubadoras sejam qualificadas em propriedade intelectual e que possuam no mínimo o Curso Geral de Propriedade Intelectual oferecido pelo Instituto Nacional da Propriedade Industrial (INPI).

\section{Referências}

ANPROTEC - ASSOCIAÇÃO NACIONAL DE ENTIDADES PROMOTORAS DE EMPREENDIMENTOS INOVADORES. Estudo, análise e proposições sobre as incubadoras de empresas no Brasil - Relatório técnico. Brasília: ANPROTEC, 2012. Disponível em: http://www. anprotec.org.br/ArquivosDin/Estudo_de_Incubadoras_Resumo_web_22-06_FINA L_pdf_59.pdf. Acesso em: $1^{\circ}$ jun. 2019.

BRASIL. Lei n. 10.973, de 2 de dezembro de 2004. Dispõe sobre incentivos à inovação e à pesquisa científica e tecnológica no ambiente produtivo e dá outras providências. Disponível em: http://www.planalto.gov.br/ccivil_03/_ato2004- 2006/2004/Lei/L10.973.htm/. Acesso em: 1 jun. 2019.

BRASIL. Lei n. 13.243, de 11 de janeiro de 2016. Dispõe sobre estímulos ao desenvolvimento científico, à pesquisa, à capacitação científica e tecnológica e à inovação. Disponível em: http://www. planalto.gov.br/ccivil_03/_ato2015-2018/2016/lei/113243.htm. Acesso em: 1 jun. 2019.

CARON, A. Inovação tecnológica e a pequenas e médias empresas local. Revista FAE BUSINESS, [S.l.], n. 8, p. 25-28, maio, 2004. Disponível em: https://img.fae.edu/galeria/ getImage/1/16570547027038246.pdf. Acesso em: 1º jun. 2019.

CARVALHO, A. C. M.; SOUZA, L. P. Ativos intangíveis ou capital intelectual: discussões das contradições na literatura e propostas para sua avaliação. Perspect. Cienc. Inf., Belo Horizonte, v. 4, n. 1, p. 73-83, jan.-jun. 1999. Disponível em: http://www.brapci.in f.br/_repositorio/2010/11/ pdf_ae1031b201_0012669.pdf. Acesso em: 10 ago. 2019.

DORNELAS, J. Empreendedorismo: transformando ideias em negócios. 6. ed. São Paulo: Atlas, 2016

INPI - INSTITUTO NACIONAL DA PROPRIEDADE INDUSTRIAL. A criação de uma marca: uma introdução às marcas de produtos e serviços para as pequenas e médias empresas. Rio de Janeiro: INPI, 2013. Disponível em: http://www.inpi.gov.br/sobr e/arquiv os/01_ cartilhamarcas_21_01_2014_0.pdf. Acesso em: $1^{\circ}$ jun. 2019.

JUNGMANN, D. de M.; BONETTI, E. A. A caminho da inovação: proteção e negócios com bens de propriedade intelectual. Guia para o empresário. Brasília: IEL, 2010. 125 p.: il. (ISBN 978-8587257-49-9). Disponível em: http://www.inpi.gov.br/sobr e/arquivos/guia_empresario_iel-senai-einpi.pdf. Acesso em: $1^{\circ}$ jun. 2019.

KRAEMER, M. E. P. Capital intelectual: a nova vantagem competitiva. Gestiopolis, [S.1.], 2004.

Disponível em: http://www.gestiopolis.com/recursos3/docs/ger/capintel.htm. Acesso em: $1^{\circ}$ jun. 2015. 
OCDE - ORGANIZAÇÃO PARA A COOPERAÇÃO E DESENVOLVIMENTO ECONÔMICO. Manual de Oslo: proposta de diretrizes para coleta e interpretação de dados sobre inovação tecnológica. 3. ed. Rio de Janeiro: Finep, 2005.

OMPI - ORGANIZAÇÃO MUNDIAL DA PROPRIEDADE INTELECTUAL. Convenção que institui a Organização Mundial da Propriedade Intelectual. Genebra: OMPI, 2002. Disponível em: https:/www.wipo.in t/edocs/pubd ocs/pt/wip o_pu b_250.pdf. Acesso em: 1 jun. 2019.

SCHUMPETER, J. A. Capitalismo, Socialismo e Democracia. Rio de Janeiro: Editora Fundo de Cultura, 1961.

SEBRAE - SERVIÇO BRASILEIRO DE APOIO ÀS MICRO E PEQUENAS EMPRESAS. Sobrevivência e mortalidade das empresas paulistas de 1 a 5 anos. São Paulo: SEBRAE, 2005.

SILVA, J. P. M. S. Incubadoras de empresas do centro-oeste: a questão da propriedade intelectual. 2016. 113f. Dissertação (Mestrado Profissional em Propriedade Intelectual e Inovação) Academia de Propriedade Intelectual, Inovação e Desenvolvimento, Rio de Janeiro, 2016.

SPOLIDORO, R. Habitats de inovação e empreendedores: agentes de transformação das estruturas sociais. TECBAHIA. Revista baiana de tecnologia, [S.l.], v. 14, n. 3, p. 9-21, 1999.

SPOLIDORO, R. et al. New models for Science and Technology Parks in response to the growing role of the cities as Innovation Habitats: perspectives from South America, Proceedings of the 30th World Conference on Science and Technology Parks, International Association of Science Parks and Areas of Innovation - IASP, Recife, Brazil, 2014.

\section{Sobre os Autores}

\section{Darlene Silveira Rodrigues}

E-mail: darlene.rodrigues@ifam.edu.br

Mestranda em Propriedade Intelectual e Transferência de Tecnologia para a Inovação, PROFNIT. Especialista em Gestão Estratégica, Inovação e Conhecimento pela Escola Superior Aberta do Brasil (2012).

Endereço profissional: Instituto Federal do Amazonas, Campus Distrito Industrial, Av. Gov. Danilo de Matos Areosa, n. 1.672, Distrito Industrial I, Manaus, AM. CEP: 69075-351.

\section{Hyrmir Alexandre Sousa}

E-mail: hyrmir_silva@yahoo.com.br

Mestranda em Propriedade Intelectual e Transferência de Tecnologia para a Inovação, PROFNIT. Universidade do Estado do Amazonas UEA (2020).

Endereço profissional: Rua das Palmeiras, n. 10, São José III, Manaus, AM. CEP: 69086-590.

\section{Raimundo Correa de Oliveira}

E-mail:rcoliveira@uea.edu.br

Doutorado em Engenharia Elétrica pela Universidade Federal de Pernambuco (2013).

Endereço profissional: Universidade do Estado do Amazonas, Escola Superior de Tecnologia, Av. Darcy Vargas, n. 1.200, Parque 10 de Novembro, Manaus, AM. CEP: 69050-020. 